\title{
Gap probability of a one-dimensional gas model
}

\author{
Y Chen ${ }^{1, \dagger}$ and S. M. Manning ${ }^{2, \dagger \dagger}$ \\ ${ }^{1}$ Department of Mathematics, Imperial College \\ 180 Queen's Gate,London SW7 2BZ UK \\ ${ }^{2}$ Department of Theoretical Physics, Oxford University \\ 1 Keble Road,OX1 3NP UK
}

December 7, 2017

\begin{abstract}
We investigate the gap formation probability of the effective one dimensional gas model recently proposed for the energy level statistics for disordered solids at the mobility edge. It is found that in order to get the correct form for the gap probability of this model, the thermodynamic limit must be taken very carefully.
\end{abstract}

$\dagger$ y.chen@ic.ac.uk

†† s.manning1@physics.oxford.ac.uk

To appear in the International Journal of Modern Physics B (1996). 


\section{Introduction}

The eigenvalue distribution of large random matrices has a long history beginning with the work of Wigner [1] on the energy level statistics of heavy nuclei. This topic has found recent applications in the theory of disordered metals, quantum chaos and string theory. The most studied and best understood of random matrix models are the Gaussian ensembles with unitary, orthogonal and symplectic symmetries [2]. Perhaps the most fundamental of all the statistical quantities of interest is the probability that an appropriately scaled interval of the spectrum is free of eigenvalues. This is a measure of the degree of correlation between the eigenvalues. There is an ansatz of Wigner which gives a simple formula that fits rather well the experimentally observed nearest levels spacing distribution [1] 1. The energy levels of disordered metals in the weak localization regime exhibit behavior which is rather well described by the correlation functions of the various Gaussian ensembles [6, 7]. Furthermore the associated level spacing distribution function compares favorably with the Wigner Ansatz, but upon increasing the strength of disorder the level spacing distribution evolves and deviates from the "standard behavior." Perhaps the most striking result is that at the mobility edge the level spacing distribution has a universal form when expressed in units of the average level spacings [8]. A random matrix model has been proposed which accounts for this continuous deviation [9]. In this model the usual unitary Gaussian ensemble is $q$ - deformed in a similar way that to the $q-$ deformation of the Laguerre ensembles [10]. The results of this ensemble give qualitative features which are in agreement with the energy level statistics obtained from numerical work on disordered solids [8]. Using the two-level correlation function obtained in [9] it was established

\footnotetext{
${ }^{1}$ Although this ansatz deviates only minutely from results of numerical experiments on random matrices, it is now known that the Wigner ansatz is not quite correct; the complete level spacing distribution functions are obtained from transcendental functions of various Painlevé types; the type depends the part of the spectrum where one performs the scaling [3, [, :5]
} 
in [11] that the number variance and the $\Delta_{3}$ statistics exhibit novel behavior. Very recently, using a combination of scaling arguments and diagrammatical analysis, it has been suggested by the authors of [13] that the density of state correlation function in the bulk of spectrum at the mobility edge behaves as $-1 /|x-y|^{2-\gamma}$ instead of $-1 /|x-y|^{2}$ in the regime of weak disorder. Here $x, y$ denotes the energy levels and $\gamma$ is related to the correlation length exponent $\nu$ and the dimension $(d)$ of the system: $\gamma=1-\frac{1}{\nu d}$.

A gas model — known as the effective plasma model in [14, 15] — with a power law pair repulsion between the particles which gives correlation function: $-1 /|x-y|^{2-\gamma}$, was proposed. Using a continuum approximation of Dyson [16], the authors of [14, 15] conclude that there is a new universal law for the level spacing distribution function for the energy levels of a disordered metal at the mobility edge. This law states that the probability that the interval $(-s, s)$ of the spectrum is free of levels behaves as $E(-s, s) \sim \mathrm{e}^{- \text {constant } s^{2-\gamma}}$, where the constant appearing here is independent of $s$.

In the continuum limit, which is expected to be valid in the limit where $N$ (the total number of particles) goes to infinity, the gas model may be described by a continuous charge density $\sigma$, following the work of Dyson [16]. In the mean field approximation this gas model has the energy functional,

$$
F[\sigma]=\frac{1}{2} \int_{J} d x \int_{J} d y \frac{\sigma(x) \sigma(y)}{|x-y|^{\gamma}}+\int_{J} d x \sigma(x) u(x), \quad 0<\gamma<1
$$

subject to

$$
\int_{J} d x \sigma(x)=N
$$

where $J$ is the support of the eigenvalues/fluid and $u(x)$ is the confining potential that holds together the repelling particles. Without loss of generality the coefficient of $1 /|x-y|^{\gamma}$ in Eq. (1) is set to unity. This is denoted by $A_{\gamma}$ in [14, 15]. Although the continuum approximation is not mathematically rigorous, previous experience suggests that it is rather robust [12]. The 
density that minimizes Eq.(1) satisfies,

$$
\int_{J} d y \frac{\sigma(y)}{|x-y|^{\gamma}}=A-u(x), \quad x \in J, \quad A=\text { Chemical potential }
$$

supplemented by $\int_{J} d x \sigma(x)=N$. At equilibrium the minimum free energy is

$$
F[J]:=F_{\text {chem }}+F_{\text {int }},
$$

where $F_{\text {chem }}:=A N / 2$ is chemical potential contribution to the free energy and

$F_{\text {int }}:=\frac{1}{2} \int_{J} d x \sigma(x) u(x)$, is the energy due to interaction between the charge distribution and the confining potential. Eq.(3) can be obtained simply by the use of the mean field equation that governs $\sigma$ and the normalization condition. Note that the separation into $F_{\text {chem }}$ and $F_{\text {int }}$ in Eq.(3) is not unique, different but equivalent expression can be found by using Eqns. (1) and (2) and the normalization condition. To obtain the density we have to deal with the inversion of the following integral equation:

$$
\int_{J} \frac{\varphi(y)}{|x-y|^{\gamma}} d y=\psi(x), \quad x \in J
$$

where $0<\gamma<1$, and $J$ is a subset of the real line. Assuming $\psi \in L^{2}[J]$, we seek $\varphi \in L^{2}[J]$. In the case where $J$ is a single interval, say $J=(0, a)$, there is a unique inversion formula,

$$
\varphi(x)=\frac{B_{\gamma}}{x^{\frac{1-\gamma}{2}}} \frac{d}{d x} \int_{x}^{a} d t \frac{t^{1-\gamma}}{(t-x)^{\frac{1-\gamma}{2}}} \frac{d}{d t} \int_{0}^{t} d s \frac{\psi(s) s^{\frac{\gamma-1}{2}}}{(t-s)^{\frac{1-\gamma}{2}}}, x \in(0, a),
$$

where

$$
B_{\gamma}:=-\frac{\Gamma(\gamma) \cos \left(\frac{\pi \gamma}{2}\right)}{\pi\left[\Gamma\left(\frac{1+\gamma}{2}\right)\right]^{2}}
$$

Eq. (4) was investigated by Carleman [17, and later by Widom in the context of stable processes [18]. This equation is also a special case of an integral equation with hypergeometric kernel [19]. However, in the many interval case,

$$
J=\cup_{p=1}^{n}\left(a_{p}, b_{p}\right), \quad a_{1}<b_{1}<\ldots<a_{n}<b_{n},
$$


no similar result has been obtained. This is an outstanding problem.

The quantity of interest, the probability, $E[J]$, that an interval $J$ is free of eigenvalues is

$$
E[J]=\frac{\left(\prod_{j=1}^{N} \int_{J^{c}} d x_{j}\right) \mathrm{e}^{-W}}{\left(\prod_{j=1}^{N} \int_{J \cup J^{c}} d x_{j}\right) \mathrm{e}^{-W}},
$$

where

$$
W=\sum_{j=1}^{N} u\left(x_{j}\right)+\sum_{1 \leq j<k \leq N} \frac{1}{\left|x_{j}-x_{k}\right|^{\gamma}},
$$

$J^{c}$ is the complement of $J$ and $J^{c} \cup J$ is the natural support of the particles.

From the previous equation we see that minus the logarithm of $E[J]$ is the change in free energy;

the free energy where all particles reside in $J^{c}$ minus the free energy where all particles reside in $J^{c} \cup J$,

$$
-\ln E[J]=\delta F:=F\left[J^{c}\right]-F\left[J \cup J^{c}\right] .
$$

The model considered in [14, 15], corresponds to the particles being distributed on the real line and and has the confining potential $u(x)=x^{2}$. The logarithm of the probability that the interval $(-a, a)$ is free of particles is formally

$$
\begin{gathered}
-\ln E[(-a, a)]=\delta F:=F[(-b,-a) \cup(a, b)]-F[(-B, B)] \\
=\frac{1}{2} N[A(a, N)-A(0, N)]+\frac{1}{2} \int_{(-b,-a) \cup(a, b)} d x \sigma(x, \operatorname{gap}) u(x)-\frac{1}{2} \int_{-B}^{B} \sigma(x) u(x),
\end{gathered}
$$

where $b(>a>0)$ is band edge of the spectrum with a gap at $(-a, a)$ and $B$ is the band edge where the spectrum is gapless. We note that with the normalization condition implemented, the respective edges $B$ and $b$ are functions of $N$, and will diverge as $N \rightarrow \infty$. Furthermore, $b$ is also a function of $a$. The deliberate use of $B$ and $b$ to distinguish the two situations is to indicate that although in the thermodynamic limit, i.e. $N \rightarrow \infty, B$ and $b$ differs by a very small amount their difference is nevertheless important and can not be neglected, for in computing $\delta F$ we are subtracting two very large numbers. Concerning the chemical potentials; $A(a, N)$ 
is the chemical potential where there is a gap and $A(0, N)$ is that without. The above remark also applies to the change in the chemical potentials. The density, $\sigma(x$, gap $)$, indicates that the integral equation must be inverted in the interval $(-b,-a) \cup(a, b)$ for which an explicit inversion formula is not known.

Although the change in the chemical potential is expected to be small as $N \rightarrow \infty$ but due to the multiplicative factor $N$; this will give a significant contribution to the change in free energy. The authors of [14, 15] assume that the change in the chemical potential (denoted by $\mu$ in 14, 15]), $\delta \mu$, is of order $s / \mathcal{E}$, (where their $s$ is our $a$ and $\mathcal{E}$ is our $B$ ) and vanishes in the thermodynamic limit, because $\mathcal{E}$ diverges as $N \rightarrow \infty$, giving negligible contribution to $\delta F$. However, further reflection suggests otherwise. From the symmetrical density given in 14, 15], $\rho(x) \sim\left(\mathcal{E}^{2}-x^{2}\right)^{\frac{1+\gamma}{2}}$, a simple calculation using the normalization condition shows that $\mathcal{E} \sim N^{\frac{1}{2+\gamma}}$, thus $\delta F_{\text {chem }} \sim N \delta \mu \sim N^{\frac{1+\gamma}{2+\gamma}} s$ and cannot be neglected. Indeed the scaling variable $\mathcal{S}$ commonly used in random matrix theory ( not the $s$ used in [14, 15]) contains the product of two quantities one of which $-s$ - tends to zero and the other a function of $N$ tends to infinity. Therefore, the appropriate scaling variable for the model studied in [14, 15] ought to be

$\mathcal{S}=N^{\frac{1+\gamma}{2+\gamma}} s$. In an example to be given below, where the problem of multi-interval inversion of the integral equation is circumvented, the meaning of the scaling variable $\mathcal{S}$ will be clarified in an exact computation within the mean field approximation. In fact we will see that the change in chemical potential is of order $a$, and is not of order $a / B$.

\section{Gap formation probability of a semi-infinite model}

As mentioned in the previous section in order to by-pass the problem of multi-interval inversion, we consider the model with $u(x)=x, x \geq 0$. We are required to compute two free energies; one with all the particles are confined in the interval $(a, b)$, where $b>a>0$, and the other with all the particles confined in $(0, B)$. Here $b$ is the band edge for a density which has a gap in $(0, a)$, 
and is supported only in $(a, b)$. The band edge $B$ is for a density that is supported over $(0, B)$. Note that when the normalization condition is implemented $b$ will be a function of $N$ and $a$ while $B$ will be a function of $N$ only. The probability that the interval $(0, a)$ has no particle in the thermodynamic limit is therefore

$$
E[\mathcal{S}]=\exp [-\delta F]
$$

where

$$
\delta F:=F[(a, b)]-F[(0, B)]
$$

The scaling variable $\mathcal{S}$ will become apparent later. To determine the free energy, we first invert the integral equation,

$$
\int_{a}^{b} d y \frac{\sigma(y)}{|x-y|^{\gamma}}=A-x, \quad a<x<b
$$

to find $\sigma$. With a shift in the variables $x \rightarrow x+a, y \rightarrow y+a$ we can now use Eq. (5) to find, for $a<x<b$,

$$
\sigma(x)=-\frac{B_{\gamma}}{(x-a)^{\frac{1-\gamma}{2}}} \frac{\Gamma^{2}\left(\frac{1+\gamma}{2}\right)}{\Gamma(\gamma)}\left[\left(A-a-\frac{(b-a)(\gamma+1)}{2 \gamma}\right) \frac{1}{(b-x)^{\frac{1-\gamma}{2}}}+\frac{1}{\gamma}(b-x)^{\frac{1+\gamma}{2}}\right] .
$$

Note that since $0<\gamma<1$ the density will have integrable divergences at $a$ and $b$. In order to have a configuration that has a lower free energy we choose

$$
A=(b-a)\left(\frac{1+\gamma}{2 \gamma}\right)+a
$$

to eliminate the divergence at $b$. This is in agreement with the general theory of integral equations with weakly singular kernel. The normalization condition, $\int_{a}^{b} d x \sigma(x)=N$, produces,

$$
N=\frac{\cos \left(\frac{\pi \gamma}{2}\right) \Gamma^{2}\left(\frac{1+\gamma}{2}\right)}{2 \pi \gamma^{2} \Gamma(\gamma)}(b-a)^{\gamma+1}
$$

From Eqns. (11) and (12), we find

$$
b=\left(\frac{N}{C_{\gamma}}\right)^{\frac{1}{\gamma+1}}+a, \quad C_{\gamma}:=\frac{\cos \left(\frac{\pi \gamma}{2}\right) \Gamma^{2}\left(\frac{1+\gamma}{2}\right)}{2 \pi \gamma^{2} \Gamma(\gamma)}
$$


and

$$
A(N, a)=\left(\frac{\gamma+1}{2 \gamma}\right)\left(\frac{N}{C_{\gamma}}\right)^{\frac{1}{\gamma+1}}+a .
$$

Eq. (13) shows that $B=\left(N / C_{\gamma}\right)^{\frac{1}{\gamma+1}}$. The first term in the r.h.s. of Eq. (14) is identified as $A(N, 0)$, the gapless chemical potential. The change in the chemical potential is therefore $a$ and not $a / B$ as claimed in [14, 15]. With $\sigma$ given by Eq. (10) and $A(N, a)$ given by Eq. (14), we compute the free energy in the presence of the gap, using elementary integration formulae,

$$
\begin{gathered}
F_{\text {int }}=\frac{N a}{2}+\frac{\Gamma^{2}\left(\frac{\gamma+3}{2}\right) \cos \left(\frac{\pi \gamma}{2}\right)}{2 \pi \gamma \Gamma(\gamma+3)}\left(\frac{N}{C_{\gamma}}\right)^{\frac{\gamma+2}{\gamma+1}}, \\
F_{\text {chem }}=\frac{\gamma+1}{4 \gamma} N\left(\frac{N}{C_{\gamma}}\right)^{\frac{1}{\gamma+1}}+\frac{N a}{2} .
\end{gathered}
$$

Therefore the free energy for which all particles are confined in $(a, b)$ is

$$
F(a, b)=N a+f_{\gamma}(N)
$$

where

$$
f_{\gamma}(N):=\frac{\gamma+1}{4 \gamma} N\left(\frac{N}{C_{\gamma}}\right)^{\frac{1}{\gamma+1}}+\frac{\Gamma^{2}\left(\frac{\gamma+3}{2}\right) \cos \left(\frac{\pi \gamma}{2}\right)}{2 \pi \gamma \Gamma(\gamma+3)}\left(\frac{N}{C_{\gamma}}\right)^{\frac{\gamma+2}{\gamma+1}} .
$$

We identify $f_{\gamma}(N)$ with $F(0, B)$, the free energy for which all particles are confined in $(0, B)$ and the change in free energy is exactly,

$$
\delta F(0, a)=F(a, b)-F(0, B)=N a .
$$

The limit which produces the scaling variable is defined as $a \rightarrow 0$ and $N \rightarrow \infty$ such that $\mathcal{S}:=N a$ is finite. Thus the probability that the interval $(0, a)$ is free of particles is

$$
E[(0, a)]=\mathrm{e}^{-\mathcal{S}}
$$

To further illustrate the meaning of $\mathcal{S}$, we consider another model with $u(x):=x^{2}, x \geq 0$. Using Eq.(5), and after some computation, we find for $a<x<b$,

$$
\sigma(x)=-\frac{\cos \left(\frac{\pi \gamma}{2}\right)}{4 \pi \gamma(\gamma+1)} \frac{1}{[(x-a)(x-b)]^{\frac{1-\gamma}{2}}}\left[C+[4 a-12 b-4(a+b) \gamma](b-x)+8(b-x)^{2}\right],
$$


where

$$
C:=-a^{2}-2 a b+3 b^{2}-4 A \gamma(\gamma+1)+4 b^{2} \gamma+(a+b)^{2} \gamma^{2}
$$

Just as in the previous example we choose

$$
A=\frac{(a+b)^{2} \gamma+3 b^{2}-a^{2}-2 a b}{4 \gamma}
$$

so that $C=0$, to eliminate the integrable singularity of $\sigma$ at $b$. Thus

$$
\sigma(x)=\frac{\cos \left(\frac{\pi \gamma}{2}\right)}{\pi \gamma(\gamma+1)} \frac{(b-x)^{\frac{1+\gamma}{2}}}{(x-a)^{\frac{1-\gamma}{2}}}[(\gamma+3) b-(1-\gamma) a-2(b-x)], \quad a<x<b .
$$

We note that the factor [...] in Eq.(24) for $a<x<b$ is greater than $(a+b)(\gamma+1)$ and is therefore positive. Application of the normalization condition leads to a transcendental equation for $b$,

$$
N=\frac{D_{\gamma} \Gamma^{2}\left(\frac{1+\gamma}{2}\right)}{2 \Gamma(\gamma+1)}(b-a)^{\gamma+2}\left[1+\frac{\gamma(b+a)}{b-a}+\frac{\gamma+1}{\gamma+2}\right], \quad D_{\gamma}:=\frac{\cos \left(\frac{\pi \gamma}{2}\right)}{\pi \gamma(\gamma+1)} .
$$

Although the explicit solution of the transcendental equation governing $b$ is not known we may nevertheless determine $b$ as a power series in $a$, which in turn can be used to calculate $F_{\text {int }}$ and $F_{\text {chem }}$, also in a power series in $a$. After some straightforward but lengthy calculations not reproduced here, we find

$$
\delta F \sim a N^{\frac{3+\gamma}{2+\gamma}}\left[1+\mathrm{O}\left(\frac{a}{N^{\frac{1}{2+\gamma}}}\right)\right] .
$$

In this case the appropriate scaling variable is

$$
\mathcal{S}:=a N^{\frac{3+\gamma}{2+\gamma}}
$$

and

$$
E[(a, b)] \sim \mathrm{e}^{-\mathcal{S}}
$$

We see that although the scaling variable $\mathcal{S}$ is the same in both cases, the thermodynamic limit is arrived in a distinct manner for distinct potentials. Following the procedure described in 15] we will find $-\ln E[s] \sim s$ for our potentials. To see how this comes about, we compute the 
change in the free energy according to the procedure given in [15] for the semi-infinite models studied above. In order to determine the probability that there are no particles in $(0, s)$, we require the change in free energy, which according to [15], is

$$
F_{s}-F_{0}=-\frac{1}{2} \int_{s}^{\infty} d x \int_{s}^{\infty} d x^{\prime} \frac{\delta \rho_{s}(x) \delta \rho_{s}\left(x^{\prime}\right)}{\left|x-x^{\prime}\right|^{\gamma}}+\frac{1}{2} \int_{0}^{s} d x \int_{0}^{s} d x^{\prime} \frac{\rho_{0}(x) \rho_{0}\left(x^{\prime}\right)}{\left|x-x^{\prime}\right|^{\gamma}}
$$

where, $\delta \rho_{s}(x)$, the change in the density due to the presence of the gap is related to the density in the absence of the gap, $\rho_{0}(x)$, via the following integral equation:

$$
\int_{s}^{\infty} d x^{\prime} \frac{\delta \rho_{s}\left(x^{\prime}\right)}{\left|x-x^{\prime}\right|^{\gamma}}=\int_{0}^{s} d x^{\prime} \frac{\rho_{0}\left(x^{\prime}\right)}{\left|x-x^{\prime}\right|^{\gamma}}
$$

Eq. (30) which governs $\delta \rho_{s}$ and which provides a determination of $F_{s}-F_{0}$ is equivalent to the statement that the change in the chemical potential vanishes in the thermodynamic limit 15]. However, as noted from the examples given above that the change of chemical potential is of order $a$. We now deduce the $s$ dependence of the gap formation probability from Eqns. (29) and (30). With the change of variables used in [15]: $z=s / x, t=s / x^{\prime}$, and $u_{g}(z)=|z|^{-2+\gamma} \delta \rho_{s}(s / z)$, Eq. (30) becomes,

$$
\int_{0}^{1} d t \frac{u_{g}(t)}{|z-t|^{\gamma}}=\frac{1}{s^{\frac{1-\gamma}{2}}} \int_{1}^{\infty} \frac{d t}{t^{\frac{3-\gamma}{2}}|z-t|^{\gamma}} .
$$

To obtain this we have used the fact the integral in the r.h.s. of Eq. (30) is dominated by $\rho_{0}(x)$ near the origin: $\rho_{0}(x) \sim 1 / x^{\frac{1-\gamma}{2}}$. Note that the $t$ integration in the r.h.s. of Eq.(31) converges. From the Eq. (31) we see that the combination $u_{g}(t) s^{\frac{1-\gamma}{2}}$ is independent of $s$. Using this, and the variables $z, t$ and $u_{g}(t)$, we find from a simple dimensional argument,

$$
F_{s}-F_{0}=\text { constant } s
$$

where the constant is independent of $s$. This suggests that the change in the free energy is independent of the confining potential $u(x)$ and universal. The statement of universality requires some qualification. If the confining potential $u(x)$ is far weaker then those considered above, the 
singularity of density near the origin, (which is determined uniquely by the confining potential via the integral equation,) will be modified and we may expect the final form of $\ln E[s]$ to be modified accordingly.

It is interesting to note that for sufficiently strong confining potentials the singularity of the

density is universal, i.e., $\rho_{0}(x) \sim 1 / x^{\frac{1-\gamma}{2}}$. This phenomena is also observed in the standard random matrix ensembles delineating the behaviour of the density for strong and weak confining potentials (no longer universal) [10, 9, 11, 12]. Thus in the situations where the singularity structure of the density is universal a universal form for $-\ln E[s]$ is found.

It appeared by adopting the two distinct approaches; one of which computes the change in the chemical potential explicitly by keeping $N$ finite but large while the other taking the limit $N \rightarrow \infty$ right off the start, we are led the same law for the gap probability, but with distinct scaling variable $\mathcal{S}=N^{\nu} a$ as opposed to $s$. It may be of interest to understand better the thermodynamic limits and clarify the meaning of $s$ and $\mathcal{S}$.

We are at present developing tools in an attempt to obtain a multi-interval inversion formula.

\section{Acknowlegement}

The authors should like to thank Mourad Ismail and Alex Hewson for helpful discussion. 


\section{References}

[1] E. P. Wigner, Proc. Camb. Phil. Soc. 47 (1951) 790.

[2] M. L. Mehta, Random Matrices (1991) (Boston, MA: Academic)

[3] M. Jimbo, T. Miwa, Y. Môri and M. Sato, Physica 1D (1980) 80.

[4] M. L. Mehta, J. de Phys. I France, 2 (1992) 1721.

[5] C. A. Tracy and H. Widom, Commun. Math. Phys. 159 (1994) 151; 161 (1994) 280 and 163 (1994) 33.

[6] B. L. Altshuler and B I Shklovskii, Sov. Phys.-JETP, 64 (1983) 127.

[7] B. L. Altshuler and A. Szafer, Phys. Rev. Lett., 70 (1993) 5879.

[8] B. I. Shklovskii, B. Shapiro, B. R. Sears, P. Lambrianides and H. B. Shore, Phys. Rev. B47(1993) 11487.

[9] K. A. Muttalib, Y. Chen, M. E. H. Ismail and V. N. Nicopoulos, Phys. Rev. Lett., 71 (1993) 471.

[10] Y. Chen, K. A. Muttalib and M. E. H. Ismail, J. Phys. C: Conds. Matter, 4 (1992) L417; J. Phys. C: Conds. Matter, 5 (1993) 177.

[11] C. Blecken, Y. Chen and K. A. Muttalib, J. Phys. A: Math. Gen. 27 (1994) L563.

[12] Y. Chen and S. M. Manning, J. Phys. A: Math. Gen. 27 (1994) 3615, Y. Chen and K. J. Eriksen, Int. J. Mod. Phys. B9 (1995) 1205, Y. Chen, K. J. Eriksen and C. A. Tracy, J. Phys. A: Math. Gen. 28 (1995) L207.

[13] V. E. Kratsov, I. V. Lerner, B. L. Altshuler and A. G. Aronov, Phys. Rev. Lett. 72 (1994) 888, A. G. Aronov, V. E. Kratsov and I. V. Lerner, Phys. Rev. Lett. 74 (1995) 1174. 
[14] A. G. Aronov, V. E. Kratsov and I. V. Lerner, JETP Lett. 59 (1994) 50.

[15] V. E. Kratsov and I. V. Lerner, J. Phys. A: Math. Gen. 28 (1995) 3623.

[16] F. J. Dyson, J. Math. Phys. 3 (1962) 140.

[17] T. Carleman, Math. Z. 15 (1922) 111.

[18] H. Widom, Trans. Am. Math. Soc. 98 (1961) 430.

[19] N. I. Akhiezer and V. A. Scherbina, Zap. matem. otd. phys.-matem. f-ta khGU and Khark. matem. o-va, 25 (1957) 191. See also, P. P. Zabreyko, A. I. Koshelev, M. A. Krasnosel'skii, S. G. Mikhlin, L. S. Rakovshchik and V. Ya. Stet'senko, Integral Equation: A Reference Text, (1975) (Leyden The Netherlands: Noordhoff) 